\title{
Measurements of core and compressed-shell temperature and density conditions in thick-wall target implosions at the OMEGA laser facility
}

\author{
R. Florido,,${ }^{1, *}$ R. C. Mancini, ${ }^{1}$ T. Nagayama,${ }^{1}$ R. Tommasini, ${ }^{2}$ J. A. Delettrez,${ }^{3}$ S. P. Regan,${ }^{3}$ and B. Yaakobi ${ }^{3}$ \\ ${ }^{1}$ Department of Physics, University of Nevada, Reno, Nevada 89557, USA \\ ${ }^{2}$ Lawrence Livermore National Laboratory, Livermore, California 94550, USA \\ ${ }^{3}$ Laboratory for Laser Energetics, University of Rochester, Rochester, New York 14623, USA
}

(Received 15 July 2010; revised manuscript received 4 April 2011; published 21 June 2011)

\begin{abstract}
A spectroscopic method is discussed to measure core and compressed-shell conditions in thick-wall plastic-shell implosions filled with deuterium and a tracer amount of argon. Simultaneous observation over a broad photon energy range of the argon line emission and the attenuation and self-emission effects of the compressed shell confining the core yields enough information to extract average temperature and density conditions in both core and compressed shell. The spectroscopic analysis also provides an estimate of the target areal density which is an important characteristic of inertial confinement fusion implosions.
\end{abstract}

DOI: 10.1103/PhysRevE.83.066408

PACS number(s): 52.25.Os, 32.30.Rj, 52.57.-z, 52.70.La

\section{INTRODUCTION}

The idea of inertial confinement fusion (ICF) is to compress a few miligrams of fuel to temperatures and densities high enough for thermonuclear fusion to proceed while mass inertia keeps the burning fuel assembled [1]. In the hot-spot ignition scheme, the target containing the thermonuclear fuel is irradiated symmetrically by high-power laser beams. The outer layers of the shell absorb radiation, heat, and ablate and, as a reaction to the outward expansion, the remaining shell is accelerated inward. At the collapse of the implosion the shell kinetic energy is converted into internal energy, compressing and heating the fuel in the core and creating a hot spot at the center that triggers the ignition [1]. Alternative approaches to ignition in which the compression and heating stages are separated have been proposed [2,3]. In particular, the shock-ignition concept relies on the slow implosion (i.e., low adiabat) of a thick-wall shell target that creates a highareal-density fuel assembly which is subsequently driven to ignition by a spherically convergent shock wave, that is, the ignitor shock [3]. This strong shock wave is launched by a sharp intensity spike at the end of the main drive pulse. The spike must be properly timed so that the ignitor shock meets the outgoing shock bouncing off the center inside the dense shell near the core. This collision originates a new shock wave moving inward that leads to further compression of the fuel and drives it to ignition. Initial shock-ignition experiments at the OMEGA laser facility [4] have successfully tested this concept [5]. Shock ignition has aroused considerable interest in the ICF community and recent studies have been performed for possible implementation at the National Ignition Facility [6] in the United States as well as at the future European High Power laser Energy Research facility [7].

In ICF experiments, diagnosis of plasma temperature and density conditions is critical to understand the performance of the implosion's hydrodynamics. In this connection, x-ray spectroscopy has proven to be a powerful and nonintrusive technique to investigate different aspects of laser-fusion

\footnotetext{
*Permanent address: Departamento de Física, Universidad de Las Palmas de Gran Canaria, E-35017 Las Palmas de Gran Canaria, Spain.
}

experiments [8] including target preheat due to fast electrons by means of $K \alpha$ emission spectroscopy $[9,10]$, average electron temperature and density in implosion cores of director indirect-drive implosions including Stark-broadened $K$ and $L$-shell line emissions [11-19], and spatial profiles of temperature and density from the analysis of $x$-ray spectra and narrow-band images [20-22].

This work aims to advance the $\mathrm{x}$-ray spectroscopy of ICF implosions by presenting a spectroscopic model and analysis for the simultaneous diagnosis of temperature and density conditions of both core and compressed shell and areal-density in thick-wall target implosions, such as those employed in the shock-ignition experiments mentioned above [5]. In addition, first spectroscopic measurements of shock-ignition implosions at the OMEGA laser facility are reported. Our method relies on the idea that a broadband spectroscopic measurement with sufficient spectral resolution can be used to extract information from several regions of a high-energy density system. In particular, for thick-wall shock-ignition implosions it employs the analysis of a tracer's x-ray spectrum over a broad range of photon energies including line features emitted in the core and attenuation due to and continuum self-emission from the compressed shell. Furthermore, the spectroscopic analysis of shock-ignition implosions presented in this work provides an estimate of the target's areal density $\rho R$ at the collapse of the implosion. This is important information for performance studies since the energy gain $G$ scales as $G \sim \theta / v_{i}^{1.25}$, where $v_{i}$ is the implosion velocity and $\theta$ represents the burn up fraction, which increases with $\rho R[3,5]$.

\section{IMPLOSION EXPERIMENTS AND SPECTROSCOPIC MODEL}

The shock-ignition implosions discussed here employed plastic shell targets that had an internal radius of $387 \mu \mathrm{m}$, a wall thickness of $40 \mu \mathrm{m}$, and an outer aluminum coating of $0.1 \mu \mathrm{m}$ for sealing purposes. They were filled with $20 \mathrm{~atm}$ of $\mathrm{D}_{2}$ and $0.072 \mathrm{~atm}$ of Ar, which was used for spectroscopic diagnosis. The capsules were irradiated by a low-adiabat pulse shape of $\sim 18.6 \mathrm{~kJ}$ UV laser energy on target composed of an 80-ps full width at half maximum (FWHM) Gaussian picket- 

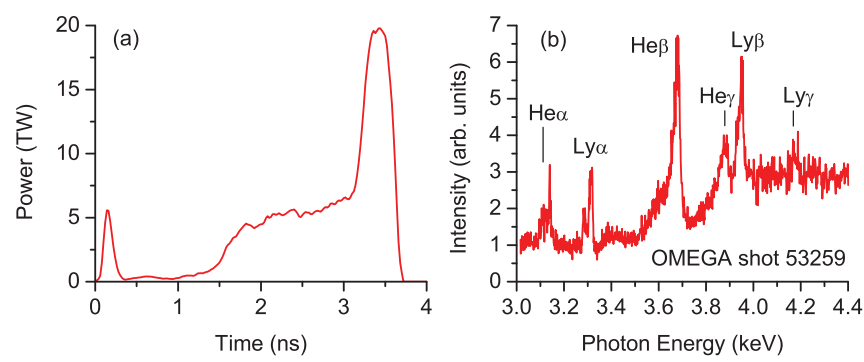

FIG. 1. (Color online) (a) Laser pulse shape. (b) Space- and timeintegrated spectrum for thick-wall target, shock-ignition implosion.

which sets the adiabat-followed by a 1.5-ns-long main drive with a sharp intensity spike at the end in order to launch the ignitor shock wave; see Fig. 1(a). The adiabat is defined as the maximum local ratio of the plasma pressure to the Fermi pressure of a degenerate electron gas at the same density and it is typically $\sim 1-2$ for the implosions discussed here. The observed argon radiation emission includes $K$-shell x-ray line transitions that span the photon energy range from 3000 to $4400 \mathrm{eV}$. An x-ray flat crystal spectrometer equipped with an Ammonium Dihydrogen Phosphate (ADP) crystal and spectral resolution power of $\lambda / \Delta \lambda \approx 1200$ was used to obtain timeand space-integrated $\mathrm{x}$-ray line spectra from the argon tracer.

An example of a time- and space-integrated spectrum recorded in the experiments is displayed in Fig. 1(b). This time-integrated measurement is characteristic of a time interval of approximately $250 \mathrm{ps}$ at the collapse of the implosion. Line transitions in $\mathrm{He}$ - and $\mathrm{H}$-like $\mathrm{Ar}$ ions are observed and labeled in the spectra, namely, He- $\alpha 1 s^{2}-1 s 2 p$, Ly- $\alpha 1 s-2 p$, He- $\beta 1 s^{2}-1 s 3 p$, He- $\gamma 1 s^{2}-1 s 4 p$, Ly- $\beta 1 s-3 p$, and Ly- $\gamma$ $1 s-4 p$. Weaker, and sometimes heavily blended with parent lines, satellite line transitions arising from autoionizing states in He- and Li-like Ar ions are also present in the data. The spectrum is significantly attenuated at low photon energies. This attenuation is so severe that the usually strong $\mathrm{He}-\alpha$ and $\mathrm{Ly}-\alpha$ lines appear among the weakest emission lines in the spectrum. This effect is interpreted in terms of the radiation absorption that takes place in the compressed shell confining the core, due to inverse bremsstrahlung (free-free) and photoionization (bound-free) processes. We emphasize that this is the first spectroscopic observation of argon tracer emission in shock-ignition implosions. In this regard, the spectrum of Fig. 1(b) shows that the signal from the argon tracer is still visible and can be used for quantitative analysis in spite of the thick-wall target employed in the experiment.

The model used to analyze the data is schematically illustrated in Fig. 2(a). It consists of a uniform sphere to characterize the state of the implosion core surrounded by a uniform concentric shell to account for the compressed shell confining the core. The parameters of the model are the core radius $R$, electron temperature $T_{c}$, electron density $N_{c}$, the compressed shell thickness $\Delta R$, electron temperature $T_{s}$, and electron density $N_{s}$. On the one hand, the value of $R$ can be computed from $N_{c}$ assuming mass conservation; we note in passing that this assumption relies on the approximation that all of the core mass contributes to the emission of radiation. On the other hand, the value $\Delta R$ is calculated from

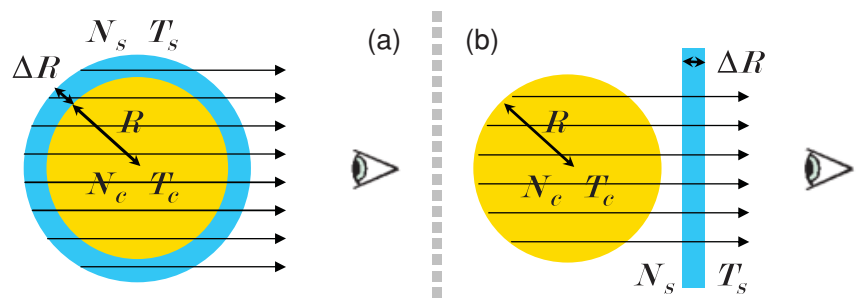

FIG. 2. (Color online) (a) Schematic illustration of the model used to analyze the data. (b) Simpler geometry with analytical solution.

the values of $R$ and $N_{s}$, as well as the fraction $\eta$ of shell mass that is imploded. The uniform model approximation for the spherical shell requires that the same imploded shell mass that contributes to the attenuation of the core radiation also contributes to the self-emission of the shell. The value of $\eta$ is estimated from LILAC one-dimensional (1D) [23] hydrodynamic simulations of the implosion. Thus, the model depends actually on four parameters: $T_{c}, N_{c}, T_{s}$, and $N_{s}$. To calculate the emergent intensity distribution for this model, we integrate the radiation transport equation for each photon energy along chords parallel to the direction of observation, and then further integrate all individual chord contributions to obtain the space-integrated spectrum for comparison with the experimental spectrum. Before comparing with data, the synthetic spectrum is convolved with the instrumental function to account for the finite spectral resolution of the instrument.

The frequency-dependent emissivity and opacity of the core and shell were calculated with population number densities from the collisional-radiative atomic kinetics model АВАKO [24]. All bound-bound, bound-free, and free-free contributions from the argon-deuterium plasma in the core and the carbon-hydrogen plasma in the compressed shell within the spectral range of the measurements were included. Energy levels and line transition rates were computed using the atomic structure code FAC [25]. For a given set of plasma temperature and density conditions, ABAKO takes into account all non-autoionizing and autoionizing states consistent with the continuum-lowering, which is estimated according to the Stewart and Pyatt model [26]. Continuum lowering is particularly important for determining the ionization balance of the compressed shell and thus the details of the contributions of photoionization and inverse bremsstrahlung to the total opacity of the compressed shell. Radiation transport effects on level population kinetics were considered via escape factors [27]. For the plasma conditions of these implosions, characteristic optical depth values $\tau$ for argon line transitions are $\tau \sim 0.5$ for the He- $\gamma$ and Ly- $\gamma$ lines, $\tau \sim 1$ for He- $\beta$ and Ly- $\beta$, and $\tau>10$ for He- $\alpha$ and Ly- $\alpha$. Detailed Stark-broadened line shapes including the ion-dynamics effect $[16,28,29]$ were used for parent and satellite transitions of the argon spectrum. For completeness, natural and Doppler line broadening were included as well, but they are small compared to Stark broadening. Additionally, a plasma broadening effect on the bound-free emissivity and opacity was included according to the approximation discussed in Ref. [30].

The model geometry shown in Fig. 2(a) was integrated numerically and employed to perform the data analysis. 
However, to gain insight into the model characteristics and the dependence on model parameters, a simplified model geometry is shown in Fig. 2(b), which corresponds to the case of a spherical plasma source (core) seen through a plasma slab (shell). The advantage of this simplified model geometry is that it has the same underlying physics of the model in Fig. 2(a) but the radiation transport equation can be integrated analytically, namely,

$$
\begin{aligned}
F^{v}= & \pi R^{2} \frac{\varepsilon_{c}^{v}}{\kappa_{c}^{v}}\left[1+\frac{e^{-2 \kappa_{c}^{v} R}}{\kappa_{c}^{v} R}-\frac{\left(1-e^{-2 \kappa_{c}^{v} R}\right)}{2\left(\kappa_{c}^{v} R\right)^{2}}\right] e^{-\kappa_{s}^{v} \Delta R} \\
& +\pi R^{2} \frac{\varepsilon_{s}^{v}}{\kappa_{s}^{v}}\left(1-e^{-\kappa_{s}^{v} \Delta R}\right),
\end{aligned}
$$

where $v$ is the photon frequency, $F^{v}$ is the emergent intensity distribution, and $\varepsilon_{c}^{v}, \kappa_{c}^{\nu}, \varepsilon_{s}^{v}$, and $\kappa_{s}^{\nu}$ stand for the temperature, density, and photon energy dependent emissivity and opacity of the core and the shell, respectively. Figure 3 graphically illustrates the details of the formation of the spectrum according to Eq. (1) for a given set of model parameters. The first term in Eq. (1) represents the radiation emitted by and transported through the core further attenuated by the transmission through the shell, that is, the product of the solid and dash traces shown in Fig. 3(a). As a result-see Fig. 3(b) - the overall magnitude of the intensity coming from the core is reduced by a factor of $\sim 10$. The second term gives the self-emission of the shell. Hence, the emergent intensity includes both the argon and the plastic emissions. Because the shell self-emission decreases with $h v$ mainly as $\exp \left(-h v / T_{s}\right)$, its effect is mostly noticeable in the low energy side of the observed photon energy range. Therefore, the spectroscopic model has the essential physics to reproduce the major spectral features observed in the recorded spectrum. Furthermore, for the analysis to be useful and practical, the model must be kept as realistic as possible but simple enough so that the information in the data is sufficient to constrain the model parameters and extract meaningful results. In this regard, a goal of this work is to show that the present model is appropriate for the analysis of space-integrated spectra collected in thick-wall implosions such as the shock-ignition implosions discussed here and that valuable information about the state of the core
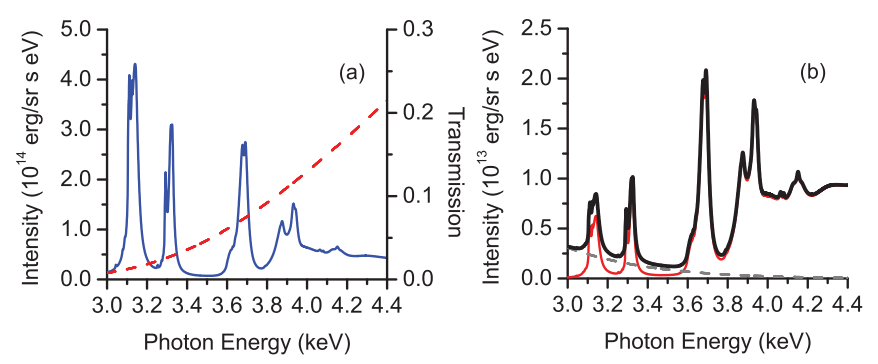

FIG. 3. (Color online) The sphere-slab model is used to illustrate the formation of the spectrum. Core and shell parameters values are $T_{s}=285 \mathrm{eV}, N_{s}=3.8 \times 10^{25} \mathrm{~cm}^{-3}, \Delta R=11 \mu \mathrm{m}, T_{c}=930 \mathrm{eV}$, $N_{c}=1.0 \times 10^{24} \mathrm{~cm}^{-3}, R=40 \mu \mathrm{m}$. (a) Core emission before being attenuated as it goes through the shell (solid line) and shell transmission factor (dashed line). (b) Attenuated core emission (thin solid line), shell self-emission (dashed line), and final emergent intensity (thick solid line). and compressed shell can be extracted from the spectroscopic analysis.

The model employed for data analysis-Fig. 2(a)contains the same physics but a more realistic spherical geometry for the compressed shell that is numerically integrated in order to compute the emergent intensity distribution to fit the experimental spectrum. Fitting the observed spectrum by means of a weighted least-squares minimization procedure yields both core and shell temperature and density conditions. Furthermore, from $N_{c}, R, N_{s}$, and $\Delta R$ the areal density $\rho R$ of the imploded target can also be obtained. To determine the best fit parameters and check their uniqueness, we performed exhaustive searches in parameter space for a given value of $\eta$. This is also important in order to test the model sensitivity on the $T_{c}, N_{c}, T_{s}$, and $N_{s}$ parameters. The $T_{c}$ and $N_{c}$ dependence is mainly determined by the argon line emission. The argon $K$-shell spectrum is sensitive to plasma electron temperature and density conditions through the temperature and density sensitivity of the atomic level population kinetics and the density dependence of the Stark-broadened line shapes. The dependence on $N_{S}$ and $T_{S}$ is brought about by the shell opacity that attenuates the line emission from the core as it goes through the compressed shell. There is also $T_{s}$ sensitivity in the continuum of the shell self-emission that becomes significant in the low photon energy end of the spectrum; that is, the slope of the underlying continuum is dependent on $T_{s}$. The overall attenuation effect of the spectrum depends also on the areal density of the compressed shell, which in turn depends on $N_{s}$ and $\Delta R$. An attenuation effect for thick-wall targets due to a high areal density shell was previously observed but only on broadband continuum emission from the core [31]. In this work, the attenuation effect is also observed on the line emission from the dopant in the core. Hence, both line and continuum emission and attenuation have to be modeled simultaneously and self-consistently to produce a good approximation to the data.

\section{RESULTS AND DISCUSSION}

The 1D hydrodynamics code LILAC was used to simulate a shock-ignition implosion with the same characteristics of the experiments performed at OMEGA. The simulation predicts that the maximum compression occurs $\sim 1$ ns after the end of the laser spike, when the core radius reaches a minimum value of $\sim 23 \mu \mathrm{m}$ and the target areal density takes a maximum value of $\sim 0.210 \mathrm{~g} \mathrm{~cm}^{-2}$. The time history of temperature and density spatial profiles computed by LILAC were postprocessed with the same atomic kinetics model used in the data analysis to compute the emergent space-integrated and either timeresolved or time-integrated spectra. From these results the space and photon energy integrated radiation power from the argon tracer can be calculated. This radiation power time history has a FWHM characteristic of $\sim 210$ ps and peaks $\sim 100$ ps before maximum compression, so that at the time of peak compression the tracer's signal is partially lost. This result suggests that the spectroscopic analysis of the time-integrated spectrum will be representative of a 200-to-250-ps time interval just before peak compression. Details of the spectral postprocessing can be found in Ref. [32]. 

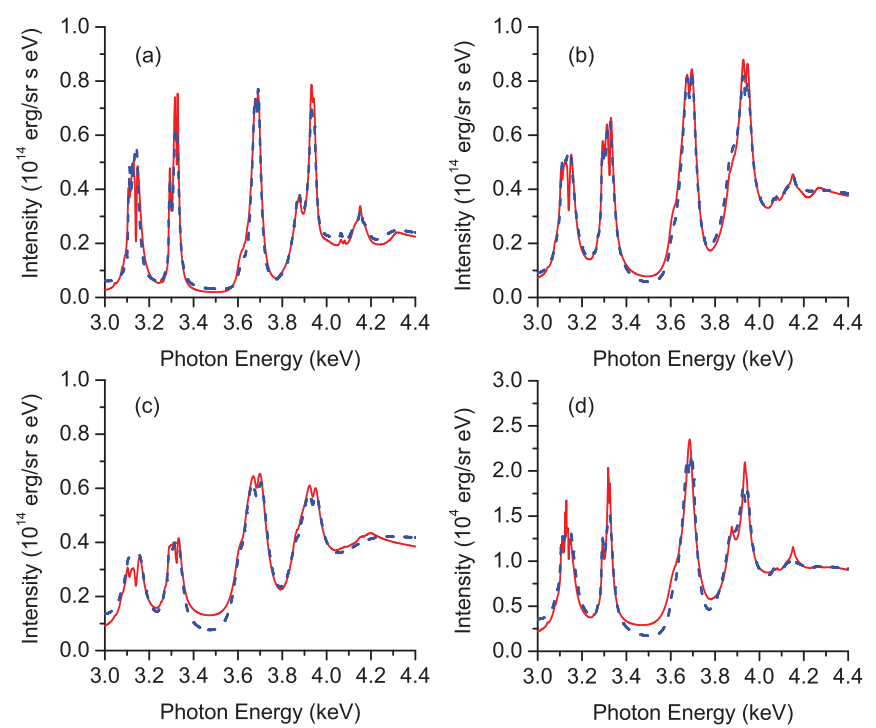

FIG. 4. (Color online) Spectroscopic analysis of synthetic data from postprocessed hydrodynamic simulation: synthetic spectra (solid lines), best model fit (dashed lines). Plots labeled (a), (b), and (c) correspond to three different time-resolved spectra; plot (d) illustrates the analysis of time-integrated synthetic spectrum. See text for details.

In order to test the analysis method, we first apply it to the analysis of several synthetic data test cases. We emphasize that the spectroscopic analysis can be applied to either timeresolved or time-integrated spectra. The results of the analysis of synthetic spectra are illustrated in Fig. 4 for three different time-resolved spectra. Spectrum (b) corresponds to the time in which the argon radiation power reaches its maximum. Spectra (a) and (c) are associated with times $60 \mathrm{ps}$ before and after the power peak, respectively. Plots labeled (a), (b), and (c) show the comparison between the synthetic spectra and model best fits. As seen in Fig. 4 good agreement was found for all cases. Since both LILAC simulations and the spectroscopic model assume spherical symmetry, differences between model fit and LILAC postprocessed spectra can be attributed to the nonuniform temperature and density spatial profiles from the hydro simulation. $\chi^{2}$ minimization was done over the entire range of photon energies from 3000 to $4400 \mathrm{eV}$. We verified that for all cases the searches in parameter space yielded a single, global minimum. However, we note that if $T_{s}$ is less than $\sim 200 \mathrm{eV}$ the sensitivity on $T_{s}$ is significantly lost and the results for $T_{s}$ and $N_{s}$ become ambiguous. Table I shows the conditions extracted from the analysis and the range of values of the simulation spatial profiles for the core and the compressed shell confining the core. The corresponding areal density values are also displayed. Mostly, the model analysis yields core and shell conditions which fall well within the range of values of LILAC spatial profiles. We note that each extracted shell density value tends to be close to the peak value of the spatial density profile. This fact is responsible in part for the small overestimate of the extracted $\rho R$ values with respect to those obtained from the hydro simulation, since the spatial extent of the compressed shell region, that is, $\Delta R$, computed by both LILAC and the spectroscopic model are similar, but the latter assumes shell uniformity. Some discrepancies between
TABLE I. Core and shell conditions extracted from the spectroscopic analysis of three-(a), (b), and (c)—-time-resolved, spaceintegrated synthetic spectra. See text for details. Ranges of temperature and density radial profiles from LILAC's simulation as well as areal density values are shown for comparison.

\begin{tabular}{llccc}
\hline \hline & & (a) & (b) & (c) \\
\hline$T_{c}(\mathrm{eV})$ & LILAC & $\sim 550-1700$ & $\sim 670-1580$ & $\sim 730-1270$ \\
& Model & 1050 & 1050 & 965 \\
$N_{c}$ & LILAC & $\sim 0.6-1.8$ & $\sim 1.3-2.8$ & $\sim 2.5-4.1$ \\
$\left(10^{24} \mathrm{~cm}^{-3}\right)$ & Model & 1.1 & 2.0 & 4.0 \\
$T_{s}(\mathrm{eV})$ & LILAC & $\sim 80-530$ & $\sim 110-645$ & $\sim 125-700$ \\
& Model & 240 & 295 & 310 \\
$N_{s}$ & LILAC & $0.2-0.8$ & $0.4-1.2$ & $0.4-1.6$ \\
$\left(10^{25} \mathrm{~cm}^{-3}\right)$ & Model & 0.9 & 1.1 & 1.2 \\
$\rho R\left(\mathrm{~g} \mathrm{~cm}^{-2}\right)$ & LILAC & 0.10 & 0.14 & 0.18 \\
& Model & 0.12 & 0.16 & 0.20 \\
\hline \hline
\end{tabular}

synthetic spectra and best model fits are observed in the photon energy region between $\mathrm{Ly}-\alpha$ and $\mathrm{He}-\beta$, particularly in case (c). In this region between line emissions differences in continuum shell emission between the postprocessed spectrum and model fit become more noticeable. We attribute these differences to the assumption that, in the model, the same amount of shell mass under uniform conditions has to contribute to both shell emissivity and opacity; however, LILAC simulation results suggest that this is not a good assumption late in time and the shell emission in postprocessed synthetic spectrum is larger than the one computed by the model.

Figure 4(d) shows the analysis of the synthetic spaceand time-integrated spectrum, that is, the synthetic spectrum equivalent to the spectrum recorded in the experiment. The discrepancies between postprocessed and model-fit spectra are more significant in the photon energy range between Ly- $\alpha$ and He- $\beta$ and between He- $\beta$ and Ly- $\beta$. In this case, the differences are due to nonuniform spatial profiles and discrepancies from later times contributing to the time-integrated spectrum. The analysis yields $T_{c}=965 \mathrm{eV}, N_{c}=1.8 \times 10^{24} \mathrm{~cm}^{-3}, T_{s}=$ $305 \mathrm{eV}, N_{s}=1.2 \times 10^{25} \mathrm{~cm}^{-3}$, and $\rho R=0.16 \mathrm{~g} \mathrm{~cm}^{-2}$. If one takes the time history of the target areal density computed by LILAC and calculates the weighted average of $\rho R$ using the argon dopant radiated power as weighting function, the result gives $\langle\rho R\rangle=0.15 \mathrm{~g} \mathrm{~cm}^{-2}$, which is very close to the one obtained from the spectroscopic analysis. This suggests that the effective core and shell conditions extracted with the spectroscopic model could be interpreted as intensity-weighted averages, similar to the emissivity-weighted averages pointed out in Ref. [33].

As stated in Sec. II, we use the LILAC simulation to estimate the fraction $\eta$ of shell mass being imploded. Therefore, we need to check the sensitivity of the analysis results to changes in $\eta$. We first checked that, according to LILAC simulation, over the $\sim 250$-ps time interval that the argon emission is on and through compression, $\eta$ is a slowly varying function of time that changes within a range of $20 \%$. Accordingly, we repeated the analysis of the time-integrated synthetic spectrum changing $\eta$ within this range. It was found that core parameters and shell temperature remain unchanged, while the shell density variation was less than $10 \%$ and the target areal density 


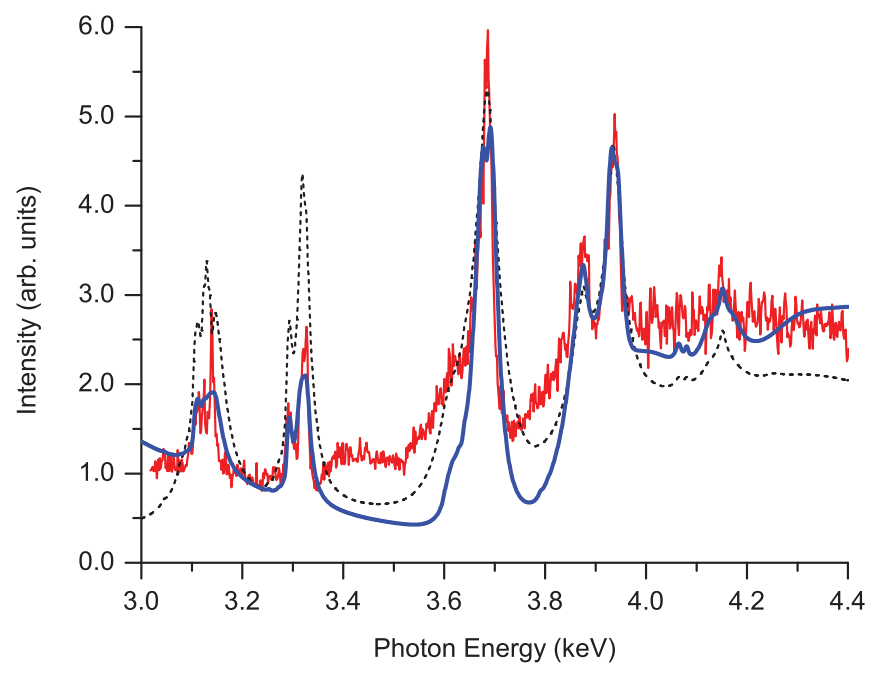

FIG. 5. (Color online) Comparison of experimental (thin solid line), postprocessed (dashed line), and best model fit (thick solid line) spectra.

changed within 5\%. Therefore, the results obtained from the spectroscopic analysis are weakly dependent on $\eta$.

Next, we analyze the space- and time-integrated experimental spectrum. The result is displayed in Fig. 5, which shows the comparison between experimental and best model fit spectra. The experimental spectrum plotted in Fig. 5 is an average of data recorded in two nominally identical OMEGA shots, that is, 53258 and 53259 , that were done to check reproducibility. The benefit of the average is that it improves the signal-to-noise ratio. To compute the uncertainties in the spectrum data points we followed the method discussed in Ref. [34]. We emphasize that the exhaustive search in parameter space for the best model fit yielded a single, global minimum characterized by a value of the normalized, weighted $\chi^{2} \sim 1$. The comparison shows disagreements for photon energies between Ly- $\alpha$ and He- $\beta$, and also between He- $\beta$ and Ly- $\beta$ lines where the model fit significantly underestimates the experimental intensity. These differences are similar to those observed in the analysis of the time-integrated synthetic spectrum, where they were attributed to the model uniformity assumption. In addition, deviations in the experiment from spherical symmetry and mixing of shell material into the core may lead to enhanced emission from satellite transitions of the $\mathrm{He}-\beta$ and $\mathrm{He}-\gamma$ lines in Li-like $\mathrm{Ar}$ as well as lower ionization stages compared to the emission calculated by the model. These contributions would come from regions located near the core's periphery which are prone to be mixed with lower-temperature shell mass and thus increase the population of lower charge ions. In this connection, we note that the Li-like satellite emission of the best (uniform) model fit on the low energy side of the $\mathrm{He}-\beta$ lines underestimates the data and that from Be-like ions is negligible.

Overall, the intensity distribution of the argon line emission, including the attenuation through the shell, is well described by the model over a photon energy range of $1.4 \mathrm{keV}$. Thus, the model incorporates the important physics to describe the core emission and the strong absorption effect in the denser and colder shell surrounding and confining the imploded core, which results in a significant attenuation of the He- $\alpha-\mathrm{Ly}-\alpha$ lines relative to the He- $\beta-\mathrm{Ly}-\gamma$ lines region. In order to perform the data analysis we proceeded in two steps. First, we excluded from the least-squares fit the photon-energy regions between Ly- $\alpha$ and $\mathrm{He}-\beta$ and between $\mathrm{He}-\beta$ and Ly- $\beta$ lines where the two-region, uniform model fails to produce a good approximation to the data. Exhaustive searches in the four-dimensional parameter space were used to find the best model fit and confirm that a single global minimum was found. The values extracted from the analysis were $T_{c}=930 \mathrm{eV} \pm 5 \%, N_{c}=1.0 \times 10^{24} \mathrm{~cm}^{-3} \pm$ $20 \%, T_{s}=285 \mathrm{eV} \pm 6 \%, N_{s}=3.8 \times 10^{25} \mathrm{~cm}^{-3} \pm 25 \%$, and $\rho R=0.17 \mathrm{~g} \mathrm{~cm}^{-2} \pm 20 \%$. The uncertainties in the parameters were computed as the standard confidence limits on estimated model parameters, which in turn result from an analysis of the curvature of the four-dimensional weighted$\chi^{2}$ surface about the minimum, taking into account the correlations between the parameters [35]. Thus, there is a $68 \%$ chance that the true parameter values fall within the given uncertainties around the measured values, which corresponds to a maximum $\chi^{2}$ variation about its minimum of $\Delta \chi^{2}=1$. Next, the analysis was repeated including the photon-energy regions between Ly- $\alpha$ and He- $\beta$ and between $\mathrm{He}-\beta$ and Ly- $\beta$ lines. In this case though the overall quality of the fits deteriorates significantly; that is, an effort to produce a better approximation to the regions between Ly- $\alpha$ and $\mathrm{He}-\beta$ and between $\mathrm{He}-\beta$ and $\mathrm{Ly}-\beta$ lines results in an overall worst fit. Furthermore, it was not possible to find a global minimum with $\chi^{2} \sim 1$. This fact is an indication of the model limitations. An extended model including a third region to characterize the mixing effect between pusher and core would improve the approximation. However, it would also require additional fitting parameters and there is not enough information in the data to determine more than four parameters.

Also shown in Fig. 5 is the time-integrated synthetic spectrum from LILAC postprocessing of Fig. 4. Compared to the best model fit, the synthetic, postprocessed spectrum shows a better agreement with the data in the region between Ly- $\alpha$ and $\mathrm{He}-\beta$, and $\mathrm{He}-\beta$ and $\mathrm{Ly}-\beta$ lines. As pointed out above, this suggests that nonuniformities are, in part, responsible for this difference with the best model fit. However, differences due to deviations from spherical symmetry and mixing not accounted for in the LILAC 1D simulation still remain. The synthetic postprocessed spectrum also shows a smaller amount of attenuation of the $\alpha$ lines compared to the experiment and an underestimation of the Ly- $\gamma$ line. This fact is consistent with the compressed shell density $N_{s}$ extracted from the experimental spectrum analysis, which is about three times larger than that from the postprocessed spectrum. The level of attenuation at a given photon energy $v$ depends on the shell optical depth $\tau_{v}$. In the uniform slab approximation, it can be written as $\tau_{v}=\frac{\kappa_{v}}{\rho_{s}}\left(\rho_{s} \Delta R\right)$, where $\rho_{s}$ is the shell mass density and $\left(\rho_{s} \Delta R\right)$ is the shell areal density, which is approximately equal to the target areal density; that is, $\left(\rho_{s} \Delta R\right) \approx \rho R$. For the high shell density values achieved in these shock-ignition implosions, the shell opacity is dominated by the free-free contribution, which in turn is proportional to $\rho_{s}^{2}$. Therefore, even though the analysis of the synthetic and experimental spectra leads to similar areal density values, the attenuation in the experimental case is more pronounced because the effective 

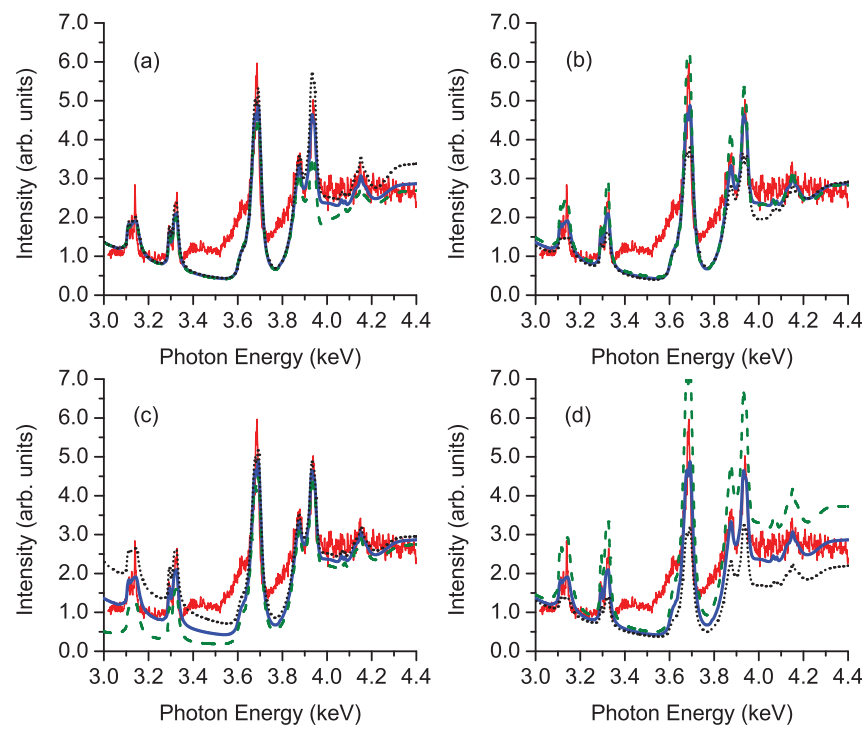

FIG. 6. (Color online) Model spectrum sensitivity to changes in each of the four fitting parameters: (a) $T_{c}$, (b) $N_{c}$, (c) $T_{s}$, (d) $N_{s}$. The thin solid lines are the experimental spectra. The dashed, thick solid, and dotted lines correspond to (a) $T_{c}=885,930$ (best fit), and $970 \mathrm{eV}$; (b) $N_{c}=0.8,1.0$ (best fit), and $1.3 \times 10^{24} \mathrm{~cm}^{-3}$; (c) $T_{s}=260,285$ (best fit) and $300 \mathrm{eV}$; and (d) $N_{s}=3.3,3.8$ (best fit), and $4.3 \times 10^{25}$ $\mathrm{cm}^{-3}$, respectively.

shell density is larger. As seen in Fig. 3, the attenuation effect is particularly severe in the region of $\mathrm{He}-\alpha$ and $\mathrm{Ly}-\alpha$, and it can even alter the ratio between these two lines. Furthermore, it can also change the intensity ratio between the $\mathrm{He}-\beta$ and Ly- $\beta$ lines.

Since the diagnosis of the core and shell conditions relies on the sensitivity of the emergent intensity distribution on the model parameters, we illustrate this dependence in the sequence of plots Figs. 6(a)-6(d). Each plot shows the change of the spectrum due to the variation of one parameter by increasing and decreasing its value within its uncertainty range. It is important to note that the change of each parameter causes a characteristic change in the spectrum. Thus, four parameters are needed to accommodate the entire spectrum and, within the model assumptions, there is enough information to uniquely determine the core and shell temperature and density conditions.

\section{SUMMARY AND CONCLUSIONS}

The main goal of this paper is to present a spectroscopic model for the simultaneous diagnosis of both core and compressed shell and areal-density conditions of thick-wall target ICF implosions. While a shell attenuation effect on broadband continuum emission from the core has been observed before [31], this work considers the effect of shell absorption on line emission from a tracer element in the core. Its photon-energy dependence changes the line intensity distribution of the tracer in a characteristic way which can be used for spectroscopic analysis. The model and analysis method were first tested with both time-resolved and time-integrated synthetic spectra obtained by postprocessing a 1D hydrodynamical simulation of the experiment, and then applied to the first spectroscopic analysis of shock-ignition implosions at the OMEGA laser facility. The data analysis of the space- and time-integrated spectrum recorded by an x-ray flat crystal spectrometer in the photon energy range from 3.0 to $4.4 \mathrm{keV}$ yields the core and compressed shell temperature and density conditions, namely, $T_{c}=930 \mathrm{eV} \pm 5 \%, N_{c}=1.0 \times 10^{24} \mathrm{~cm}^{-3} \pm 20 \%$, $T_{s}=285 \mathrm{eV} \pm 6 \%, N_{s}=3.8 \times 10^{25} \mathrm{~cm}^{-3} \pm 25 \%$. The analysis is based on a weighted $\chi^{2}$ minimization procedure and uncertainties in the parameters were determined from a confidence interval statistical study that takes into account the correlations between model parameters. Discrepancies between the best model fit and the experimental spectrumparticularly in the region between $\mathrm{Ly}-\alpha$ and $\mathrm{He}-\beta$ and between $\mathrm{He}-\beta$ and Ly- $\beta$-may be attributed to spatial nonuniformities and deviations from spherical symmetry and mixing that cannot be accounted for by the model, since it assumes uniform core and shell regions. Despite this fact, the model contains the essential ingredients to reproduce the major spectral features observed in the experimental spectrum and overall it describes well the intensity distribution of the argon line emission, including the attenuation through the shell, over a photon energy range of $1.4 \mathrm{keV}$. Furthermore, the spectroscopic analysis also provides an estimate of $\rho R=$ $0.17 \mathrm{~g} \mathrm{~cm}^{-2} \pm 20 \%$ for the target's areal density at the collapse of the implosion. We note in closing that initial thickwall shock-ignition experiments performed at OMEGA [5] reported areal density values, inferred from the analysis of the energy distribution of secondary protons, of $\sim 0.18 \mathrm{~g} \mathrm{~cm}^{-2}$ and $\sim 0.16 \mathrm{~g} \mathrm{~cm}^{-2}$ for optimized and nonoptimized spike pulse shapes, respectively, and using undoped targets filled with 25 atm of $D_{2}$. The spectroscopic analysis of the shock-ignition experiments discussed here produced areal density values consistent with the results of those early experiments based on particle diagnostics. However, the spectroscopic analysis also yielded the temperature and density conditions of the core and compressed shell which are important characteristics of the fuel assembly at the collapse of the implosion, thus providing a more complete picture of the conditions achieved in the shock-ignition experiment.

\section{ACKNOWLEDGMENTS}

This work was supported by DOE/NLUF Grant DE-FG5209NA29042 and LLNL. R. Florido has also been supported by Spanish Ministry of Science and Innovation Grant No. ENE2009-11208 and the Keep-in-Touch Project of the EU.
[1] S. Atzeni, Plasma Phys. Controlled Fusion 51, 124029 (2009).

[2] M. Tabak, J. Hammer, M. E. Glinsky, W. L. Kruer, S. C. Wilks, J. Woodworth, E. M. Campbell, and M. D. Perry, Phys. Plasmas 1, 1626 (1994).
[3] R. Betti, C. D. Zhou, K. S. Anderson, J. L. Perkins, W. Theobald, and A. A. Solodov, Phys. Rev. Lett. 98, 155001 (2007).

[4] T. R. Boehly et al., Opt. Commun. 133, 495 (1997).

[5] W. Theobald et al., Phys. Plasmas 15, 056306 (2008). 
[6] L. J. Perkins, R. Betti, K. N. LaFortune, and W. H. Williams, Phys. Rev. Lett. 103, 045004 (2009).

[7] X. Ribeyre, G. Schurtz, M. Lafon, S. Galera, and S. Weber, Plasma Phys. Controlled Fusion 51, 015013 (2009).

[8] S. P. Regan et al., High Energy Density Phys. 5, 234 (2009).

[9] B. Yaakobi, I. Pelah, and J. Hoose, Phys. Rev. Lett. 37, 836 (1976).

[10] B. Yaakobi, C. Stoeckl, W. Seka, J. A. Delettrez, T. C. Sangster, and D. D. Meyerhofer, Phys. Plasmas 12, 062703 (2005).

[11] B. Yaakobi, D. Steel, E. Thorsos, A. Hauer, and B. Perry, Phys. Rev. Lett. 39, 1526 (1977).

[12] B. Yaakobi, S. Skupsky, R. L. McCrory, C. F. Hooper, H. Deckman, P. Bourke, and J. M. Soures, Phys. Rev. Lett. 44, 1072 (1980).

[13] C. J. Keane, B. A. Hammel, A. L. Osterheld, and D. R. Kania, Phys. Rev. Lett. 72, 3029 (1994).

[14] H. Nishimura, T. Kiso, H. Shiraga, T. Endo, K. Fujita, A. Sunahara, H. Takabe, Y. Kato, and S. Nakai, Phys. Plasmas 2, 2063 (1995)

[15] C. F. Hooper Jr., D. P. Kilcrease, R. C. Mancini, L. A. Woltz, D. K. Bradley, P. A. Jaanimagi, and M. C. Richardson, Phys. Rev. Lett. 63, 267 (1989).

[16] D. A. Haynes Jr., D. T. Garber, C. F. Hooper Jr., R. C. Mancini, Y. T. Lee, D. K. Bradley, J. Delettrez, R. Epstein, and P. A. Jaanimagi, Phys. Rev. E 53, 1042 (1996).

[17] N. C. Woolsey et al., Phys. Rev. E 56, 2314 (1997).

[18] S. P. Regan, J. A. Delettrez, R. Epstein, P. A. Jaanimagi, B. Yaakobi, V. A. Smalyuk, F. J. Marshall, D. D. Meyerhofer, and W. Seka, Phys. Plasmas 9, 1357 (2002).

[19] R. Florido, T. Nagayama, R. C. Mancini, R. Tommasini, J. A. Delettrez, S. P. Regan, V. A. Smalyuk, R. Rodríguez, and J. M. Gil, Rev. Sci. Instrum. 79, 10E310 (2008).
[20] I. Golovkin et al., Phys. Rev. Lett. 88, 045002 (2002).

[21] L. Welser-Sherrill et al., Phys. Rev. E 76, 056403 (2007).

[22] T. Nagayama, R. C. Mancini, R. Florido, R. Tommasini, J. A. Koch, J. A. Delettrez, S. P. Regan, V. A. Smalyuk, L. A. Welser-Sherrill, and I. E. Golovkin, Rev. Sci. Instrum. 79, 10E921 (2008).

[23] J. Delettrez, R. Epstein, M. C. Richardson, P. A. Jaanimagi, and B. L. Henke, Phys. Rev. A 36, 3926 (1987).

[24] R. Florido, R. Rodríguez, J. M. Gil, J. G. Rubiano, P. Martel, E. Mínguez, and R. C. Mancini, Phys. Rev. E 80, 056402 (2009).

[25] M. F. Gu, Can. J. Phys. 86, 675 (2008).

[26] J. C. Stewart and K. D. Pyatt, Astrophys. J. 144, 1203 (1966).

[27] R. C. Mancini, R. F. Joyce, and C. F. Hooper Jr., J. Phys. B 20, 2975 (1987)

[28] L. A. Woltz and C. F. Hooper Jr., Phys. Rev. A 38, 4766 (1988).

[29] R. C. Mancini, D. P. Kilcrease, L. A. Woltz, and C. F. Hooper Jr., Comput. Phys. Commun. 63, 314 (1991).

[30] E. J. Iglesias and H. R. Griem, J. Quant. Spectrosc. Radiat. Transfer 55, 383 (1996).

[31] B. Yaakobi, R. Epstein, and F. J. Marshall, Phys. Rev. A 44, 8429 (1991); F. J. Marshall, J. A. Delettrez, R. Epstein, and B. Yaakobi, Phys. Rev. E 49, 4381 (1994).

[32] R. Florido, R. C. Mancini, T. Nagayama, R. Tommasini, J. A. Delettrez, S. P. Regan, and B. Yaakobi, Rev. Sci. Instrum. 81, 10E307 (2010).

[33] I. E. Golovkin and R. C. Mancini, J. Quant. Spectrosc. Radiat. Transfer 65, 273 (2000).

[34] R. L. Coldwell and G. J. Bamford, The Theory and Operation of Spectral Analysis Using ROBFIT (American Institute of Physics, College Park, MD, 1991).

[35] W. H. Press, S. A. Teukolsky, W. T. Vetterling, and B. P. Flannery Numerical Recipes: The Art of Scientific Computing, 3rd ed. (Cambridge University Press, Cambridge, 2007). 